FOUNDATIONS OF COMPUTING AND DECISION SCIENCES Vol. 40

\title{
ON THE INDEPENDENCE NUMBER OF SOME STRONG PRODUCTS OF CYCLE-POWERS
}

\author{
Marcin JURKIEWICZ, Marek KUBALE, Krzysztof OCETKIEWICZ *
}

\begin{abstract}
In the paper we give some theoretical and computational results on the third strong power of cycle-powers, for example, we have found the independence numbers $\alpha\left(\left(C_{10}^{2}\right)^{\otimes 3}\right)=30$ and $\alpha\left(\left(C_{14}^{4}\right)^{\otimes 3}\right)=14$. A number of optimizations have been introduced to improve the running time of our exhaustive algorithm used to establish the independence number of the third strong power of cycle-powers. Moreover, our results establish new exact values and/or lower bounds on the Shannon capacity of noisy channels.
\end{abstract}

Keywords: strong product, exhaustive search algorithm, independence number, Shannon capacity

\section{Introduction and basic definitions}

The values of the independence numbers of the strong products of graphs are strictly related to the problem of calculation the Shannon capacity of a noisy channel and its characteristic graph. The problem was posed by Shannon [9]. He found the capacities of small graphs on $n \leq 5$ vertices, except the value of this measure for the cycle on 5 vertices. Twenty three years later Lovász [8] proved that the Shannon capacity of the cycle on 5 vertices is equal to $\sqrt{5}$. But after a half century, we still do not know the Shannon capacity of odd cycles on $n \geq 7$ vertices.

In the paper we present some theoretical observations allowing for better assessment of the Shannon capacity of a noisy channel. We have established new exact values of the Shannon capacity of some classes of noisy channels from the known result on the channels corresponding to the cycle on 5 vertices. We also present results of computational experiments that take advantage of these observations and provide

* Gdansk University of Technology, Faculty of Electronics, Telecommunications and Informatics, Department of Algorithms and System Modelling, Gabriela Narutowicza 11/12, 80-233 Gdansk, Poland, \{marcin.jurkiewicz, kubale, krzysztof.ocetkiewicz\}@eti.pg.gda.pl 
exact solutions for some special cases, for example, we have found the independence numbers $\alpha\left(\left(C_{10}^{2}\right)^{\otimes 3}\right)=30$ and $\alpha\left(\left(C_{14}^{4}\right)^{\bigotimes 3}\right)=14$.

We begin by introducing definitions needed in the rest of the paper. In particular we give a formal definition of the Shannon capacity. For a deeper discussion of this subject we refer the reader to $[7,9,8]$.

Let $G=(V(G), E(G))$ be a finite undirected graph. A set of vertices $S$ of $G$ is said to be an independent set of vertices if they are pairwise nonadjacent. The independence number of $G$, denoted by $\alpha(G)$, is defined to be the size of a largest independent set of $G$.

In this paper we use the following operations on graphs.

Definition 1.1. Given a graph $G$ and a positive integer $k$. The $k$-power $G^{k}$ of $G$ is the graph on the same set $V(G)$ in which two vertices are adjacent if and only if they have the distance at most $k$ in $G$.

Definition 1.2. Given two graphs $G=(V(G), E(G))$ and $H=(V(H), E(H))$, the strong product $G \otimes H$ is defined as follows. The vertices of $G \otimes H$ are all pairs of the Cartesian product $V(G) \times V(H)$. There is an edge between $\left(v, v^{\prime}\right)$ and $\left(w, w^{\prime}\right)$ if and only if one of the following holds:

- $\{v, w\} \in E(G)$ and $\left\{v^{\prime}, w^{\prime}\right\} \in E(H)$,

- $v=w$ and $\left\{v^{\prime}, w^{\prime}\right\} \in E(H)$,

- $v^{\prime}=w^{\prime}$ and $\{v, w\} \in E(G)$.

We write $G^{\bigotimes n}$ to denote $G \otimes G \otimes \cdots \otimes G$, where $G$ occurs $n$ times.

For a discrete noisy channel $\Gamma$ with an input alphabet $A$, we define the following notion. The characteristic graph of a channel $\Gamma$ is a graph $G=(V, E)$ such that the vertex set $V=A$ and $\{v, w\} \in E$ if and only if $S_{v} \cap S_{w} \neq \emptyset$, where $S_{v}$ is the set of letters attainable on output, when there is $v$ on input.

Baumert et al. [3] and independently Hales [6] established the exact formula on the strong product of two cycles. Recently, Badalyan and Markosyan [2] generalized this result to the strong product of cycle-powers. Because we are interested in calculating the Shannon capacity, we present their results under the assumption that the same $n$-vertex cycle $C_{n}$ appears in the strong product.

Theorem 1.1 (Badalyan and Markosyan [2]). Given an arbitrary cycle $C_{n}$ and a positive integer $k$, we have

$$
\alpha\left(C_{n}^{k} \otimes C_{n}^{k}\right)=\left\lfloor\frac{n}{k+1}\left\lfloor\frac{n}{k+1}\right\rfloor\right\rfloor,
$$

where $C_{n}^{k}$ is the $k$-th power of $C_{n}$.

Recently, Bachoc et al. [1] have found some new results on the so-called Lovász number for cycle-powers, which are strongly related to the considered problem. Let $G$ be a graph of order $n$, and let $\mathcal{A}_{G}$ be the set of real symmetric matrices $A$ indexed 
by $V(G)$ that satisfy $A_{v w}=1$, if $v=w$ or if $v$ and $w$ are non-adjacent. The Lovász number $\vartheta(G)$ is defined as

$$
\vartheta(G)=\inf _{A \in \mathcal{A}_{G}} \lambda_{1}(M)
$$

where $\lambda_{1}(M)$ denotes the largest eigenvalue of $M[8]$. It is well known that

$$
\alpha\left(G^{\bigotimes i}\right) \leq \vartheta^{i}(G)
$$

for any positive integer $i$.

In 1956, in his seminal paper [9], Shannon introduced the notion of a capacity of a noisy channel in a reliable transmission.

Definition 1.3. The Shannon capacity of a discrete noisy channel $\Gamma$ with the characteristic graph $G$ is defined as

$$
\Theta(G)=\sup _{n} \sqrt[n]{\alpha\left(G^{\bigotimes n}\right)}
$$

The next theorem follows immediately from the definition of the Shannon capacity.

Theorem 1.2 (Shannon [9]). Let $G$ be a graph and let $i$ be a positive integer. Then

$$
\Theta(G) \geq \sqrt[i]{\alpha\left(G^{\bigotimes i}\right)}
$$

It turns out that there exist graphs for which $\Theta(G)>\alpha(G)$. From the information theory point of view, the graphs fulfilling the sharp inequality describing channels facilitate an advantage in the zero-error communication [7].

It is interesting that the exact value of the Shannon capacity of $C_{7}$ is still unknown. Vesel and Žerovnik showed that $3.2237 \leq \Theta\left(C_{7}\right) \leq 3.3176$ [10].

\section{Some theoretical aspects}

In this section we will present some theoretical facts that we have used as the first approximation in our computational experiments.

Note that an independent set of the strong products of a cycle-power $\left(C_{n}^{k}\right)^{\bigotimes p}$ can be represent by the packing of $p$-dimensional cubes of side $k+1$ into the $n \times n \times \cdots \times n$ torus, where $n$ occurs $p$ times (see Fig. 1).

Using this interpretation of an independent set of $\left(C_{n}^{k}\right)^{\bigotimes p}$, we get the following observations.

Observation 2.1. Given a cycle $C_{n}$ and positive integers $k, p$, we have

$$
\alpha\left(\left(C_{n+1}^{k}\right)^{\otimes p}\right) \geq \alpha\left(\left(C_{n}^{k}\right)^{\bigotimes p}\right)
$$

Observation 2.2. Given a cycle $C_{n}$ and positive integers $k, p, q$, we have

$$
\alpha\left(\left(C_{q n}^{(k+1) q-1}\right)^{\bigotimes p}\right)=\alpha\left(\left(C_{n}^{k}\right)^{\bigotimes p}\right)
$$

and

$$
\Theta\left(C_{q n}^{(k+1) q-1}\right)=\Theta\left(C_{n}^{k}\right) .
$$



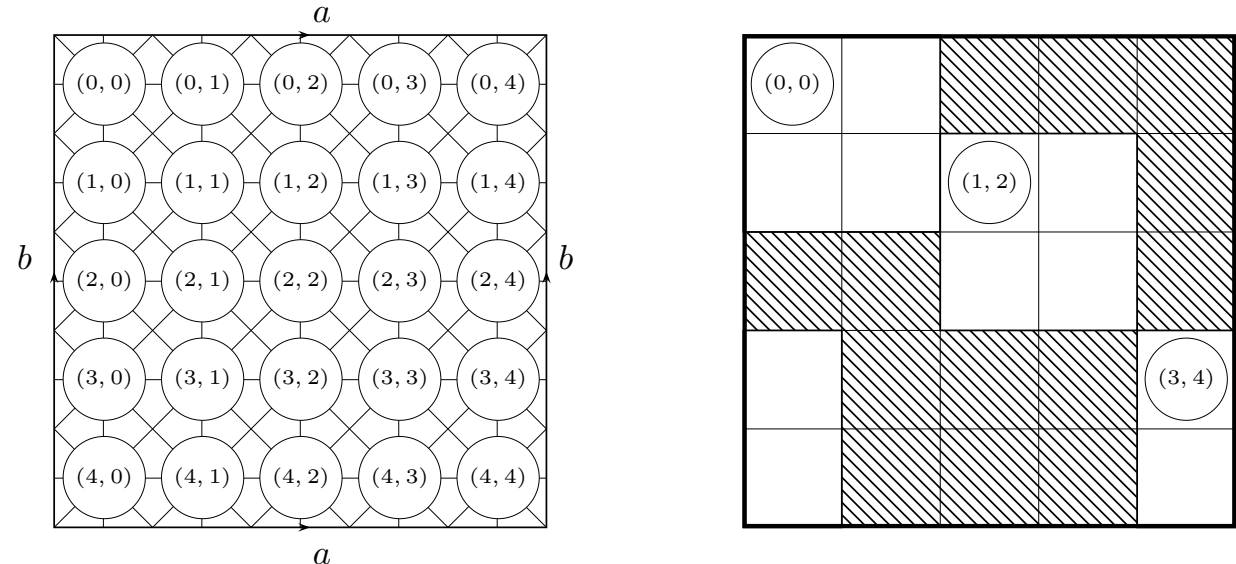

Figure 1: On the left-hand side we present graph $\left(C_{5}^{1}\right)^{\bigotimes 2}$. The graph is embedded in the torus represented as the square in which opposite sides are identified. On the right hand-side we show an independent set $\{\{0,0\},\{1,2\},\{3,4\}\}$ and the corresponding packing. The independent vertices are placed in upper-left corners of 2-dimensional cubes of side 2 , hence are not adjacent to each other. It is easy to generalize this idea to $p$-dimensional cubes of side $k+1$.

For example, from $\alpha\left(\left(C_{5}^{1}\right)^{\bigotimes 3}\right)=10, \alpha\left(\left(C_{7}^{1}\right)^{\bigotimes 3}\right)=33$ and the above observations, we obtain $\alpha\left(\left(C_{15}^{5}\right)^{\bigotimes 3}\right)=10, \alpha\left(\left(C_{14}^{3}\right)^{\otimes 3}\right)=33$, respectively. The old and new results on $\left(C_{n}^{k}\right)^{\bigotimes 3}$ are gathered in Table 1 of the next section. Moreover, from $\Theta\left(C_{5}\right)=\sqrt{5}[8]$ and the equality (8), we have $\Theta\left(C_{5 i}^{2 i-1}\right)=\sqrt{5}$ for $i \in \mathbb{N}$.

The next proposition gives another upper bound on the independence number of the strong product of cycle-powers and it is an extension of the result due to Baumert et al. [3].

Proposition 2.3. For a given cycle $C_{n}$ and positive integers $k, p$ such that $k<n$, we have

$$
\alpha\left(\left(C_{n}^{k}\right)^{\bigotimes p}\right) \leq\left\lfloor\frac{n}{k+1} \alpha\left(\left(C_{n}^{k}\right)^{\bigotimes(p-1)}\right)\right\rfloor,
$$

where $\alpha\left(\left(C_{n}^{k}\right)^{\bigotimes 0}\right)=1$.

Proof. Notice that a packing of $p$-dimensional cubes of side $k+1$ into the $n^{p}$-torus may be considered as the composition of $n$ packings of $(p-1)$-dimensional cubes of side $k+1$ into the $n^{p-1}$-torus. Also, remember that a $p$-dimensional cube and an $n^{p}$-torus are composed of $(k+1)^{p}$ and $n^{p}$ cells, respectively. Because a maximum packing into the $n^{p}$-torus consists smaller (or the same) number of cells than $n$ maximum packings into the $n^{p-1}$-torus, so

$$
(k+1)^{p} \alpha\left(\left(C_{n}^{k}\right)^{\bigotimes p}\right) \leq n(k+1)^{p-1} \alpha\left(\left(C_{n}^{k}\right)^{\bigotimes(p-1)}\right) .
$$

We divide both sides of the above inequality by $(k+1)^{p}$, and the proof is complete. 
Corollary 2.4. Given a cycle $C_{n}$ and positive integers $k, p$ such that $(k+1) \mid n$, we have

$$
\alpha\left(\left(C_{n}^{k}\right)^{\bowtie p}\right)=\left(\frac{n}{k+1}\right)^{p}
$$

Proposition 2.3 gives us, for example, the following inequalities $\alpha\left(\left(C_{13}^{2}\right)^{\bigotimes 3}\right) \leq 73$ and $\alpha\left(\left(C_{19}^{3}\right)^{\bigotimes 3}\right) \leq 90$.

\section{Computational experiments}

The following results were obtained by using an exhaustive search algorithm. The state of the algorithm was encoded within a 3 -dimensional cube of size $n^{3}$ representing the state of each vertex - either it belonged to the neighborhood of the current independent set or not (see Fig. 2).

Each vertex was assigned its coordinates $(x, y, z)$, where $0 \leq x, y, z \leq n-1$. As the value of $n$ did not exceed 32 in any test, the whole row of the cube (all vertices with the same coordinates $y$ and $z$ ) could be stored in one machine word (using one bit per vertex).

A number of optimizations have been introduced to improve the running time of the algorithm. To reduce the overhead of creating a new state for the next level in search tree, each row had a stack associated to it and before changing the value of the row, previous content was pushed onto stack. Restoring the previous state required retrieving previous content of the modified rows from stack. This method reduced the number of copied machine words to create the new state from $n^{2}$ to $(2 k+1)^{2}$.

The second improvement to the algorithm was added to reduce the size of search tree. We used the fact that any vertex at given level $z$ (a level contains all vertices with the same coordinate $z$ ) affects only up to $k$ levels above and $k$ levels below this level. Since we are filling our cube in the top-down order (from $z=0$ to $n-1$ ) the bits that have impact on computations on the following levels of the search tree are

\begin{tabular}{|c|c|c|c|c|c|c|}
\hline 10 & 1 & 12 & $\mathrm{Le}$ & Level 4 & Level 5 & evel 6 \\
\hline 1110011 & 111 & 1110011 & 0000000 & 0000000 & 1110011 & 1110011 \\
\hline 1110011 & 111 & 011 & 0000000 & 0000000 & 1110011 & 1110011 \\
\hline 1110011 & 1110011 & 1110011 & 0000000 & 0000000 & 1110011 & 1110011 \\
\hline 0000000 & 0000000 & 0000000 & 0000000 & 0000000 & 0000000 & 0000000 \\
\hline 0000000 & 0000000 & 0000000 & 0000000 & 0000000 & 0000000 & 0000000 \\
\hline 1100011 & 1100011 & 1100011 & 0000000 & 0000000 & 1100011 & 1100011 \\
\hline 1100011 & 1100011 & 1100011 & 0000000 & 0000000 & 1100011 & 1100011 \\
\hline
\end{tabular}

Figure 2: Contents of the cube $\left(C_{7}^{2}\right)^{\bigotimes 3}$ (for $n=7$ and $k=2$ ) with the vertex $(0,0,0)$ added. The value 1 represents positions that are forbidden, the value 0 means that a vertex can be added to the independent set at this place. 
those that occupy current level and $k$ levels below the current level (in other words, only $k$ previous levels are important, as these are the only levels that contain vertices that influence the current bit setup). Therefore, for a given configuration of bits in level $z$ and $k$ following levels, the number of vertices that the algorithm was able to add to the independent set on levels $z, \ldots, n-1$ was remembered and any time the same configuration of bits was observed on levels $z, \ldots, z+k$ (all indexing arithmetic is done modulo $n$ ), the stored value was retrieved. If the current independent set enlarged by the retrieved value was not greater than the best known independent set size, the branch of the search tree was cut (Steps 16-22 in Algorithm 1).

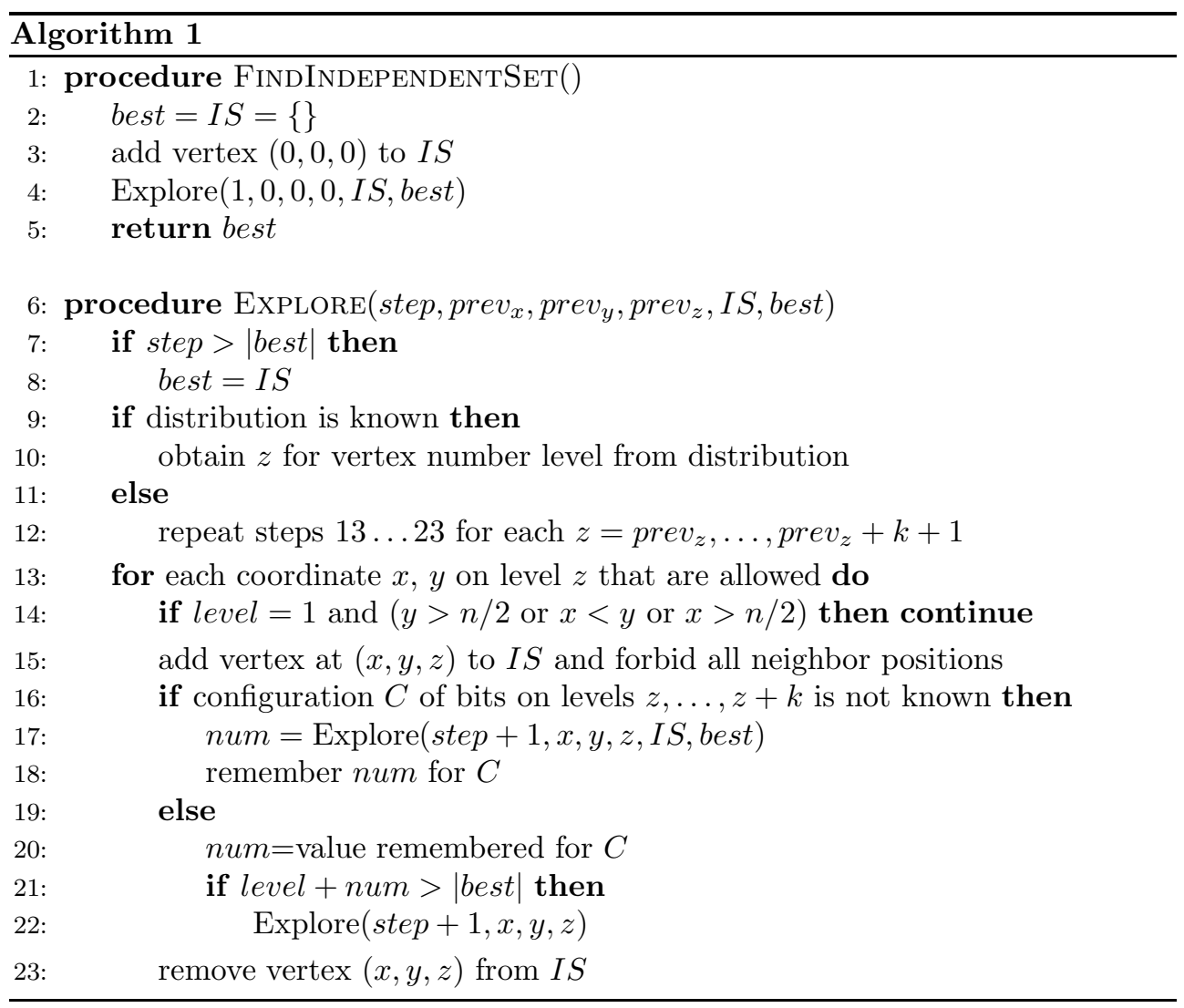

Another observation that was employed allowed us to remove some of symmetric cases. The first vertex was always placed at position $(0,0,0)$. The second one could only occupy positions $(x, y, z)$ where $x \leq n / 2$ and $x \leq y \leq n / 2$, since all other configurations of those two coordinates can be obtained by symmetric reflections and translations (Step 14 in Algorithm 1). Finally, for some problems we calculated allowed distributions, as follows. For a given independent set, create a vector of length $n$, where the $i$-th element is equal to the number of vertices with coordinate $z$ equal to $i$. Such a vector will be called a distribution. Since every level of our cube represents 
a copy of $\left(C_{n}^{k}\right)^{\bigotimes 2}$, the number of vertices with the same $z$ coordinate cannot exceed $\alpha\left(\left(C_{n}^{k}\right)^{\bigotimes 2}\right)$. Moreover, since vertex on level $i$ affects all levels from $i-k$ to $i+k$, the total number of vertices on levels $i-k, \ldots, i+k$ cannot exceed $\alpha\left(\left(C_{n}^{k}\right)^{\bigotimes 2}\right)$, for $i=0, \ldots, n$. Knowing the value of $\alpha\left(\left(C_{n}^{k}\right)^{\bigotimes 2}\right)$ and assuming a number of vertices in the independent set, we can compute all possible distributions of these vertices. For example, given the problem $\left(C_{2}^{11}\right)^{\bigotimes 3}$ with 40 vertices, the only possible distribution is $\{3,4,3,4,4,3,4,4,3,4,4\}$, with respect to rotations and reversion of the sequence.

When allowed distributions were computed, they were used to determine $z$ coordinates of each vertex. For example, knowing that the distribution is $\{3,4,3,4,4$, $3,4,4,3,4,4\}$, we know that three first vertices should have $z$ coordinate equal to 0 , another four vertices should have $z=1$ and so on. When there were more than one possible distributions, all of them were tested. When distribution was not given, the maximal allowed difference of the $z$ coordinate between two consecutive vertices was not allowed to exceed $k+1$ (Step 12 in Algorithm 1). In cases when the number of possible distributions was large, distributions were not used since using them would not improve the running time of the algorithm.

All the results took about 100 hours of computation on a quad-core Intel i5-3550 3.3GHz. They are summarized in Table 1.

Table 1: Exact values and bounds for $\left(C_{n}^{k}\right)^{\bigotimes 3}$. The previously known values are placed in the second column and are taken from [3, 5, 4]. Symbols: a - Algorithm 1, o - Observation 2.1, o' - Observation 2.2, p - Proposition 2.3, c - Corollary 2.4 and $\vartheta$ - Inequality (3).

\begin{tabular}{|c|c|c|c|c|c|c|}
\hline$n \backslash^{k}$ & $\mathbf{1}$ & $\mathbf{2}$ & $\mathbf{3}$ & $\mathbf{4}$ & $\mathbf{5}$ & $\mathbf{6}$ \\
\hline $\mathbf{4}$ & 8 & 1 & 1 & 1 & 1 & 1 \\
\hline $\mathbf{5}$ & 10 & 1 & 1 & 1 & 1 & 1 \\
\hline $\mathbf{6}$ & 27 & $8^{c}$ & 1 & 1 & 1 & 1 \\
\hline $\mathbf{7}$ & 33 & $8^{a}$ & 1 & 1 & 1 & 1 \\
\hline $\mathbf{8}$ & 64 & $12^{a}$ & $8^{c}$ & 1 & 1 & 1 \\
\hline $\mathbf{9}$ & 81 & $27^{c}$ & $8_{a}^{\vartheta}$ & 1 & 1 & 1 \\
\hline $\mathbf{1 0}$ & 125 & $30^{a}$ & $10^{o^{\prime}}$ & $8^{c}$ & 1 & 1 \\
\hline $\mathbf{1 1}$ & 148 & $40 p$ & $13_{a}^{p}$ & $8_{o}^{p}$ & 1 & 1 \\
\hline $\mathbf{1 2}$ & 216 & $64^{c}$ & $27^{c}$ & $8^{a}$ & $8^{c}$ & 1 \\
\hline $\mathbf{1 3}$ & 247 & $73 p$ & $29 p$ & $10^{a}$ & $8_{o}^{p}$ & 1 \\
\hline $\mathbf{1 4}$ & 343 & $84{ }^{2} p$ & $33^{o^{\prime}}$ & $14^{a}$ & $8^{a}$ & $8^{c}$ \\
\hline $\mathbf{1 5}$ & 390 & $125^{c}$ & $\begin{array}{c}41 p \\
382\end{array}$ & $27^{c}$ & $10^{o^{\prime}}$ & $8_{o}^{p}$ \\
\hline
\end{tabular}

In particular, the newly found independent sets and lower bounds on the Shannon capacity (Theorem 1.2) are as follows: 
- $\alpha\left(\left(C_{7}^{2}\right)^{\bigotimes 3}\right)=8$ (the set of size 9 does not exist) $\{(0,0,0),(3,0,0),(0,3,0),(3,3,0),(0,0,3),(3,0,3),(0,3,3),(3,3,3)\}$,

- $\alpha\left(\left(C_{8}^{2}\right)^{\bigotimes 3}\right)=12$ (the set of size 13 does not exist) $\{(0,7,5),(1,2,1),(2,6,0),(2,4,5),(3,7,3),(3,1,6),(4,3,2),(5,6,6)$, $(6,2,7),(6,0,2),(7,5,1),(7,3,4)\}$, $\Theta\left(\left(C_{8}^{2}\right)^{\otimes 3}\right) \geq 2.2894$

- $\alpha\left(\left(C_{10}^{2}\right)^{\bigotimes 3}\right)=30$ (the set of size 31 does not exist) $\{(0,0,0),(3,0,0),(1,3,1),(9,7,1),(8,4,2),(2,6,2),(7,1,3),(4,2,3)$, $(5,5,3),(6,8,3),(3,9,3),(0,1,4),(9,8,4),(1,4,5),(8,5,5),(3,0,6)$, $(7,2,6),(4,3,6),(5,6,6),(2,7,6),(6,9,6),(0,1,7),(9,8,7),(1,4,8)$, $(8,5,8),(7,2,9),(4,3,9),(5,6,9),(2,7,9),(6,9,9)\}$

- $\alpha\left(\left(C_{10}^{3}\right)^{\bigotimes 3}\right)=10$ (the set of size 11 does not exist)

$\{(0,0,0),(4,2,1),(8,4,3),(0,0,5),(4,2,5),(2,6,5),(6,8,5),(8,4,7)$, $(2,6,9),(6,8,9)\}$,

- $\alpha\left(\left(C_{11}^{3}\right)^{\bigotimes 3}\right)=13$ (equal to the upper bound) $\{(0,0,0),(4,2,0),(8,4,1),(1,7,2),(5,9,3),(1,3,4),(5,5,5),(9,8,6)$, $(6,1,7),(2,10,7),(10,4,8),(3,6,9),(7,8,10)\}$, $\Theta\left(\left(C_{11}^{3}\right)^{\bowtie 3}\right) \geq 2.3513$

- $\alpha\left(\left(C_{12}^{4}\right)^{\bigotimes 3}\right)=8$ (the set of size 9 does not exist) $\{(0,0,0),(5,0,0),(0,5,0),(5,5,0),(0,0,5),(5,0,5),(0,5,5),(5,5,5)\}$,

- $\alpha\left(\left(C_{13}^{4}\right)^{\bigotimes 3}\right)=10$ (the set of size 11 does not exist) $\{(0,0,0),(5,0,0),(0,5,0),(5,5,2),(0,0,5),(10,5,5),(7,10,5)$, $(5,2,7),(2,7,7),(7,7,10)\}$

- $\alpha\left(\left(C_{14}^{4}\right)^{\bigotimes 3}\right)=14$ (equal to the upper bound) $\{(0,6,9),(1,9,4),(2,12,13),(3,1,8),(4,4,3),(5,7,12),(6,10,7)$, $(7,13,2),(8,2,11),(9,5,6),(10,8,1),(11,11,10),(12,0,5),(13,3,0)\}$, $\Theta\left(\left(C_{14}^{4}\right)^{\otimes 3}\right) \geq 2.4101$

- $\alpha\left(\left(C_{14}^{5}\right)^{\bigotimes 3}\right)=8$ (the set of size 9 does not exist) $\{(0,0,0),(6,0,0),(0,6,0),(6,6,0),(0,0,6),(6,0,6),(0,6,6),(6,6,6)\}$,

- $10 \leq \alpha\left(\left(C_{16}^{5}\right)^{\otimes 3}\right) \leq 12$ (the set of size 13 does not exist) $\{(0,0,0),(6,0,2),(2,6,2),(8,8,4),(12,2,6),(0,12,6),(2,4,8)$, $(6,14,8),(8,8,10),(0,10,12)\}$

- $\alpha\left(\left(C_{20}^{6}\right)^{\bigotimes 3}\right)=14$ (equal to the upper bound) $\{(0,0,1),(7,3,2),(14,8,4),(1,11,5),(8,16,7),(15,1,8),(2,4,9),(9,9,11)$, $(16,14,12),(3,17,14),(10,2,15),(17,7,17),(4,10,18),(11,15,19)\}$, $\Theta\left(\left(C_{20}^{6}\right)^{\bigotimes 3}\right) \geq 2.4101$. 


\section{Acknowledgement}

This article was partially supported by the Narodowe Centrum Nauki under grant DEC-2011/02/A/ST6/00201.

\section{References}

[1] Bachoc, C., PÊcher, A., And Thiéry, A. On the theta number of powers of cycle graphs. Combinatorica 33, 3 (2013), 297-317.

[2] Badalyan, S. H., and Markosyan, S. E. On the independence number of the strong product of cycle-powers. Discrete Math. 313, 1 (2013), 105-110.

[3] Baumert, L. D., Mceliece, R. J., Rodemich, E., Rumsey, Jr., H. C., Stanley, R., AND TAYlor, H. A combinatorial packing problem. In Computers in algebra and number theory (Proc. SIAM-AMS Sympos. Appl. Math., New York, 1970). Amer. Math. Soc., Providence, R.I., 1971, pp. 97-108. SIAM-AMS Proc., Vol. IV.

[4] Bohman, T., Holzman, R., and Natarajan, V. On the independence numbers of the cubes of odd cycles. Electron. J. Combin. 20, 3 (2013), Paper 10, 19.

[5] Codenotti, B., Gerace, I., And Resta, G. Some remarks on the Shannon capacity of odd cycles. Ars Combin. 66 (2003), 243-257.

[6] Hales, R. S. Numerical invariants and the strong product of graphs. J. Combinatorial Theory Ser. B 15 (1973), 146-155.

[7] Körner, J., And Orlitsky, A. Zero-error information theory. IEEE Trans. Inform. Theory 44, 6 (1998), 2207-2229. Information theory: 1948-1998.

[8] Lovász, L. On the Shannon capacity of a graph. IEEE Trans. Inform. Theory 25, 1 (1979), 1-7.

[9] Shannon, C. E. The zero error capacity of a noisy channel. Institute of Radio Engineers, Transactions on Information Theory, IT-2, September (1956), 8-19.

[10] Vesel, A., AND ŽERovniK, J. Improved lower bound on the Shannon capacity of $C_{7}$. Inform. Process. Lett. 81, 5 (2002), 277-282.

Received 6.10.2014, accepted 11.02.2015 